\section{High-density lipoprotein- cholesterol, reverse cholesterol transport, and cardiovascular risk: a tale of genetics?}

\author{
Giovanni Cimmino, Chiara D'Amico, \\ Giovanni Ciccarelli, Marco Golino, \\ Alberto Morello, Saverio D'Elia, \\ Valeria Marchese, Paolo Golino \\ Department of Cardio-Thoracic \\ and Respiratory Sciences, Section \\ of Cardiology, Second University \\ of Naples, Italy
}

\section{Abstract}

Cholesterol deposition plays a central role in atherogenesis. The accumulation of lipid material is the result of an imbalance between the influx and efflux of cholesterol within the arterial wall. High levels of plasma low-density lipoprotein-cholesterol are considered the major mechanism responsible for the influx and accumulation of cholesterol in the arterial wall, while high-density lipoprotein (HDL) cholesterol seems responsible for its efflux. The mechanism by which cholesterol is removed from extra-hepatic organs and delivered to the liver for its catabolism and excretion is called reverse cholesterol transport (RCT). Epidemiological evidence has associated high levels of HDL-cholesterol/ApoA-I with protection against atherosclerotic disease, but the ultimate mechanism(s) responsible for the beneficial effect is not well established. HDLs are synthesized by the liver and small intestine and released to the circulation as a lipid-poor HDL (nascent HDL), mostly formed by ApoA-I and phospholipids. Through their metabolic maturation, HDLs interact with the ABCAl receptor in the macrophage surface increasing their lipid content by taking phospholipids and cholesterol from macrophages becoming mature HDL. The cholesterol of the HDLs is transported to the liver, via the scavenger receptor class B, type I, for further metabolization and excretion to the intestines in the form of bile acids and cholesterol, completing the process of RCT. It is clear that an inherited mutation or acquired abnormality in any of the key players in RCT mat affect the atherosclerotic process

\section{Introduction}

Despite tremendous advancements in therapeutic strategies in the last 20 years, cardio- vascular disease (CVD) are still the number one cause of mortality and morbidity worldwide, with atherosclerosis and its thrombotic complications being responsible for most of CVD deaths. ${ }^{1}$ Atherosclerosis is a diffuse process characterized by the lipids deposition and other blood-borne material within the arterial wall of almost all vascular territories. On one hand, low-density lipoprotein-cholesterol (LDL-C) penetrates into the subendothelial space as a consequence of endothelial dysfunction (which is considered the very initial step of atherosclerosis), where it can be oxidized (oxLDL-C); oxLDL-C is highly toxic, and as part of a mechanism of defense, it is phagocytized by the vessel wall macrophages. Furthermore, oxLDL-C triggers a series of proinflammatory reactions via different mediators, perpetuating the activation and recruitment of monocytes-macrophages and other inflammatory cells. Macrophages, engulfed by lipid material, ultimately become foam cells, which may undergo apoptotic death, release cholesterol to the vessel wall and, more importantly, inflammatory substances like tissue factor and metalloproteinases, thus making atherosclerotic lesions more prone to rupture. On the other, high-density lipoprotein-cholesterol (HDL-C) is essential in cholesterol efflux from the arterial wall (Figure 1) and its transport to the liver for further metabolism and final excretion via the intestine (Figure 2), a process called reverse cholesterol transport (RCT). Thus, lipid accumulation within the arterial wall may be the result of an imbalance between cholesterol influx by LDL-C and efflux by HDL-C. ${ }^{2}$

\section{Cardiovascular disease risk reduction and high-density lipoprotein-cholesterol}

In many epidemiological studies high levels of HDL-C are associated with a reduced risk of CVD, while low HDL-C levels linked to an increased risk. ${ }^{3}$ Conversely, it has been shown that the incidence of HDL-C below $35 \mathrm{mg} / \mathrm{dL}$ was $\approx 3$-fold higher among men with premature $(<60$ years of age) coronary heart disease than in age-matched controls. ${ }^{4}$ In addition, a post-hoc analysis of the TNT study ${ }^{5}$ showed that HDL-C levels in patients receiving statins were predictive of major cardiovascular events (MACE) across the study cohort, both when HDL-C was considered as a continuous variable and when subjects were stratified according to quintiles of HDL-C. Even among study subjects with LDL-C levels below $70 \mathrm{mg} / \mathrm{dL}$, those in the highest quintile of HDL-C levels were at less risk for MACE than those in the lowest quintile (Figure 3). However, recent
Correspondence: Paolo Golino, Department of Cardio-Thoracic and Respiratory Sciences, Section of Cardiology, Second University of Naples, A.O. S. Anna e S. Sebastiano, via Tescione 1,81100 Caserta, Italy.

Tel. +39.0823.306395 - Fax: +39.0823 .232395 .

E-mail:paolo.golino@unina2.it

Key words: atherosclerosis, high-density lipoproteins, low-density lipoproteins, reverse cholesterol transport.

Acknowledgements: Giovanni Cimmino would like to thank Juan Jose Badimon, Director of the Atherothrombosis Research Unit, Cardiovascular Institute, Mount Sinai Hospital, New York City for all the learning experiences and invaluable guidance in this field and Borja Ibanez, from CNIC, Madrid, Spain, for all the valuable help.

Received for publication: 16 April 2013. Revision received: 15 July 2013.

Accepted for publication: 22 July 2013.

This work is licensed under a Creative Commons Attribution NonCommercial 3.0 License (CC BYNC 3.0).

(C) Copyright G. Cimmino et al., 2013

Licensee PAGEPress, Italy

Cardiogenetics 2013; 3:e7

doi:10.4081/cardiogenetics.2013.e7

human studies evaluating two different cholesterol ester transfer protein inhibitors (torcetrapib and dalcetrapib) have cast some doubt on the good cholesterol HDL hypothesis. Despite a significant increase in HDL-C levels no beneficial cardiovascular outcomes was reported. ${ }^{6}$ However, abundant evidence in animals models show that increased HDL is associated with decreased atherosclerosis progression or even regression, thus a new theory has surfaced, that HDL-C levels may not be an ideal predictor of cardiovascular risk, rather HDL-C function may be a better indicator.?

In this review, we will discuss one of the major functions of HDL, namely its role in promoting RCT, along with several genetic mechanisms by which RCT can become dysfunctional, and new clinical evidence linking HDL-C functionality to cardiovascular outcomes.

\section{Reverse cholesterol transport and high-density lipoprotein- cholesterol functions}

The atheroprotective properties of HDL-C (i.e. antioxidant, anti-inflammatory, endothelial cell maintenance functions, mediator of RCT) are well known. Although these activities have not been directly compared, it is likely 
that RCT plays a crucial role in anti-atherogenic effect of HDL-C. The RCT hypothesis proposes that HDL-C accepts cholesterol from the periphery, such as arterial wall cells, and deliver it to the liver for excretion via the bile salts. In 1990 the pioneering work of Badimon et al. employed HDL-C as a therapeutic agent: it was the first preclinical evidence that plaque regression is feasible, showing that HDL-C infusion regressed pre-existing lesions in a rabbit model of atherosclerosis. ${ }^{8}$ Other HDL-Celevating interventions corroborated this finding in animals ${ }^{9}$ and in humans. ${ }^{10}$

HDLs are a heterogeneous group of particles, different in size, shape, density, cholesterol and phospholipid content, as well as in apolipoprotein composition. The life cycle of HDL begins with apolipoprotein A-I (ApoA-I) being synthesized by the liver and, after interaction with hepatic ATP-binding cassette transporter 1 (ABCA1), is secreted into plasma as lipid-poor ApoA-I. The maturation process starts by acquisition of cholesterol and phospholipids (PLs) via ABCA1-mediated efflux from the liver and the transfer of cholesterol, PLs, and apolipoproteins from chylomicrons and vey low-density lipoproteins (VLDL) during lipoprotein-lipase-mediated lipolysis to form nascent pre--HDLs. Additional cholesterol and PLs is acquired from cells in extrahepatic tissues via ABCA1-mediated efflux, progressively generating more cholesterolenriched particles. The enzyme lecithin-cholesterol acyltransferase (LCAT), carried on HDLs, esterifies the free cholesterol (FC) to cholesteryl ester (CE), which migrate to the core of the HDL-particle to form mature HDLs that can acquire additional lipid via ABCG1 and SR-BI-mediated efflux ${ }^{2}$ (Figure 1).

Thus, genetic variants in any of the key players in RCT may be of great importance for cholesterol accumulation and/or removal, ultimately modifying the atherosclerotic process.

\section{ATP binding cassette family}

The ATP binding cassette (ABC) family is a large conserved family of transmembrane proteins, which require energy (ATP) to transport substrates from the cytoplasm out of the cell or into specific intracellular organelles against concentration gradients. This family have been subdivided into either full or half transporters and into 7 subgroups (A-G), based on sequence similarity and domain organization. ${ }^{11}$ Type Al is ubiquitously expressed in humans and promotes efflux of PLs and FC from cells to lipid-poor ApoA-I. It is essential for normal lipidation of lipid-poor ApoA-I as well as for regulation of apoptosis and inflammation. In its functional absence ApoA-I is rapidly catabolized and it does not reach maturity.

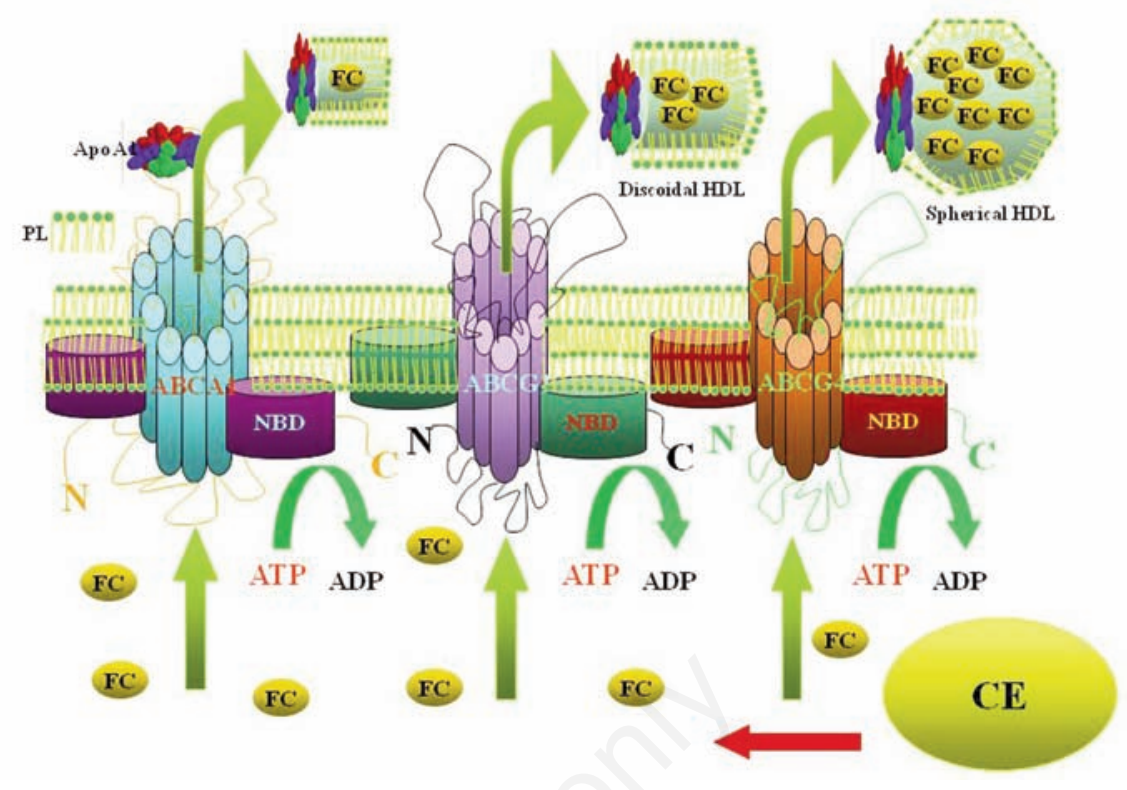

Figure 1. High-density lipoprotein (HDL) particles start out as apolipoproteins produced by the liver, called apoA1. Precursor molecules are released in HDL called pre-B-HDL, incorporating small quantities of cholesterol and lipids, especially phospholipids (PL). ATP-binging cassette transports (ABC) proteins transport various molecules across extraand intra-cellular membranes. Cholesterol from non-hepatic peripheral tissues is transferred to HDL by the ABCA1. ABCG1 and ABCG4 are necessary for the further lipidation required becoming spherical particles HDL. The free cholesterol (FC) is converted to cholesteryl esters (CE) by the enzyme lecithin-cholesterol acyltransferase (LCAT).

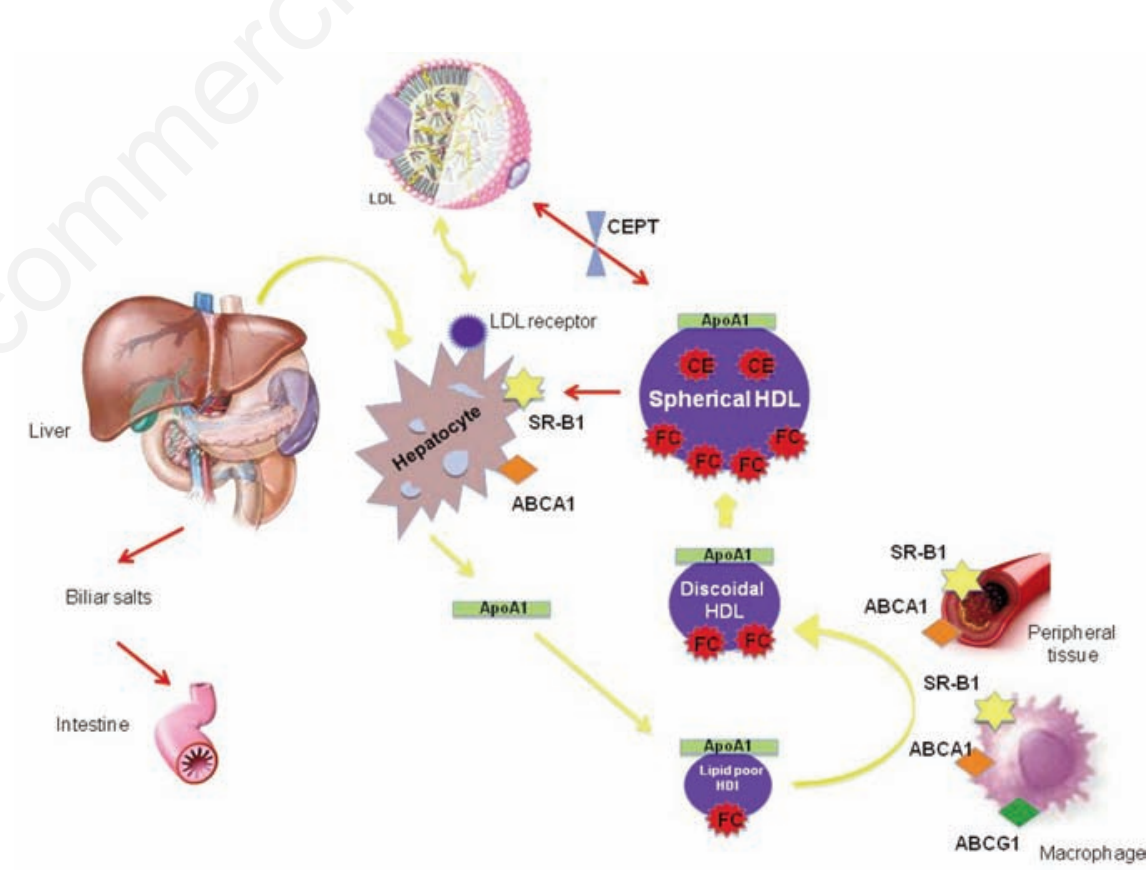

Figure 2. Schematic view of reverse cholesterol transport. Apia-I is responsible for the metabolic fate of high-density lipoprotein (HDL) cholesterol HDLs and it is synthesized by the liver. HDLs are released to the circulation as a lipid-poor HDL (nascent HDL), mostly formed by Apia-I and phospholipids (PL). Through their metabolic maturation, HDLs interact with the ABCA1, ABCG1 and also SR-B1 receptor in the macrophage surface and peripheral tissue increasing their lipid content by taking PLs and cholesterol becoming spherical HDL. The cholesterol of the HDLs is transported to the liver, via the scavenger receptor class $\mathrm{B}$, type I (SR-BI), for further metabolization and excretion to the intestines in the form of bile acids and cholesterol, completing the process of reverse cholesterol transport. 
In Tangier disease a complete loss of function mutations in $A B C A 1$ gene was found, with virtually absence of HDL-C and very low levels of ApoA-I, as well as cholesterol accumulation in peripheral macrophage-enriched tissue. ${ }^{12}$

To date, more than 100 mutations in $A B C A 1$ gene have been identified, most of which are misses. The latest described affects the acceptor splice site of intron 20 assessing its impact on pre-mRNA splicing..$^{13}$

Animal studies indicate that ABCAl and ABCG1 play a key role in facilitating cholesterol efflux and RCT. Mice deficient in ABCA1 or ABCG1 have a marked increase in foam-cell accumulation and atherosclerosis. ${ }^{14}$ Furthermore, heterozygosity for an ABCA1 mutation (K776N) confers two- to three-fold risk of CVD. This mutation is localized in the middle of the ABCA1 protein in a domain predicted to be transmembrane or very close to the extracellular surface; functionality of this area is still unknown. This residue is of particular interest because: i) is completely conserved between species; ii) the amino acid substitution results in a change in side chain charge (basic to uncharged polar); iii) $\mathrm{K} 776 \mathrm{~N}$ is relatively frequent in Caucasians (3/1000); iv) diseasecausing mutations have been identified in the corresponding region of a closely related gene, the cystic fibrosis transmembrane conductance regulator. ${ }^{15}$

A very large pool of $\mathrm{FC}$ is the adipose tissue that it's known to support cholesterol efflux to HDL/ApoA-I in vitro. In contrast to liver and macrophages, adipocytes cholesterol efflux is controlled by ABCA1 and SR-BI, but not ABCG1, and is suppressed by some inflammatory adipocytokines. ${ }^{16}$

ABCG proteins are unique half transporters with the $\mathrm{ABC}$ domain localized to the aminoterminal side of the transmembrane part. Homodimers G1:G1 and G4:G4 and heterodimer G5:G8 have a role in transporting sterols across membranes. ${ }^{11}$ Early studies showed that macrophages express high levels of ABCG1 mRNA when converted to lipidloaded foam cells by incubation with modifiedLDL or specific oxysterols, or following the induction of the nuclear receptor liver-X-receptor. ABCG1 is highly expressed in multiple tissues (i.e. lung, brain, kidney, spleen) while it is low/undetectable in hepatocytes and enterocytes, suggesting that, unlike ABCA1, ABCG1 has no role in lipid absorption or lipoprotein secretion.

The physiological importance of ABCG1 was revealed upon analysis of $\mathrm{ABCG1}^{- \text {-LacZ }}$ knockin mice. Surprisingly, the major phenotype involved massive lipid accumulation in the lungs of these mice, particularly in alveolar macrophages and, to a lesser extent, the surfactant-secreting type- 2 cells. This deposition was age dependent, accelerated by a high-fat diet, and so severe that the lungs turned white, with chronic inflammation, macrophage accumulation, lymphocyte infiltration, and elevated cytokines levels, cytokine receptors and matrix remodeling enzymes. This was a result in part of the impaired ability of ABCG1 ${ }^{-}$pulmonary macrophages to efflux surfactant-derived cholesterol. ${ }^{17}$ Lipidation of ApoE-containing lipoproteins in glial cells in the brain is also ABCG1 dependent. ${ }^{18}$ Mice knock-out for ABCG1/ABCA1 demonstrated that loss of both transporters resulted in an even more striking lipid-accumulation phenotype in macrophages and specific tissues than in single knock-out mice. ${ }^{19}$

ABCG1 is highly expressed in endothelial cells, where it mediates cholesterol efflux to exogenous HDL in vitro and, as recently reported, it balances vasoconstriction/vasodilation by expression of active endothelial nitric oxide synthase. ${ }^{20}$

Unclear results come from animal studies involving $\mathrm{ABCG}^{-}{ }^{-}$mice, that are not hypercholesterolemic and, consequently, do not develop spontaneous atherosclerosis. ${ }^{21}$ It has been observed that transplantation of bone marrow cells from $\mathrm{ABCG1}^{-}$mice into hypercholesterolemic LDLR $^{--}$atherosclerosis-prone mice ${ }^{22}$ induced a moderately significant increase in lesion $\operatorname{size}^{23}$ as well as significant decreases (20-50\%) of plaque. ${ }^{22}$ These paradoxical results were attributed to increased susceptibility of the macrophages to apoptosis or to an increase in $\mathrm{ABCAl}$ expression and secretion of $\mathrm{apoE}^{22}$ from $\mathrm{ABCG1}{ }^{-1}$ macrophages. Moreover, unexpected data came also from transgenic mice where over expression of human ABCG1 in either $\mathrm{LDLR}^{-/}$or $\mathrm{ApoE}^{-/-}$hypercholesterolemic mice did not attenuate atherosclerosis development. ${ }^{23}$

Finally, it has been also displayed that ABCA1, ABCG1, and HDL inhibit the proliferation of hematopoietic stem and multipotential progenitor cells and connect expansion of these populations with leukocytosis and accelerated atherosclerosis. $^{24}$

\section{Scavenger receptor $\mathbf{B} \mathbf{I}$}

SR-BI is the membrane HDL receptor, encoded by $S C A R-B I$ gene. It mediates cholesterol transfer to/from HDL (direction depends on cholesterol gradient) and also the selective uptake of other lipids (i.e. CE, PLs, triglycerides), thus promoting depletion of HDL-C core lipids. In mammals, liver is the only organ capable to synthesize/metabolize cholesterol; thus, cholesterol transport to the liver is essential for its removal from the body. HDL-C is transferred to the hepatocyte via SR-BI, for further metabolization and excretion into the intestine in the form of bile acids and cholesterol, completing the process of RCT. During its transport to the liver, mature HDLs can transfer part of its CE to VLDL-C and LDL-C via cholesteryl ester transfer protein (CETP). ${ }^{2}$

Mutations, deletions and other abnormali-

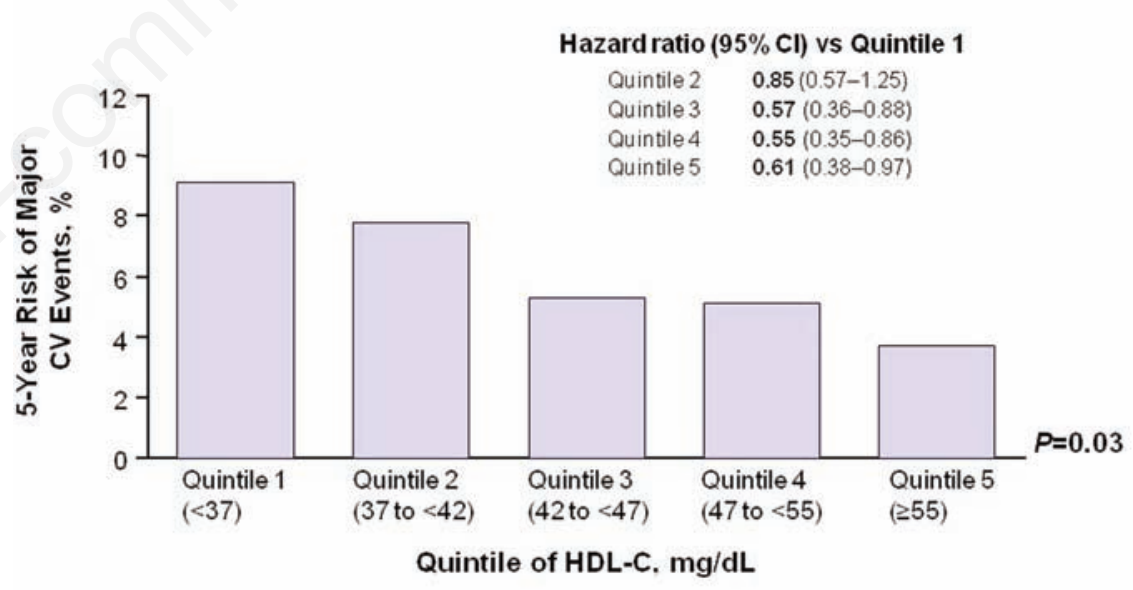

Figure 3. A post-hoc analysis of the TNT trial showing that high levels of high-density lipoprotein-cholesterol (HDL-C) significantly reduced the 5-year risk of major CV events, even in patients with low-density lipoprotein-cholesterol (LDL-C) $(<70 \mathrm{mg} / \mathrm{dL})$. Using multivariate analysis, the authors explored whether HDL-C levels are clinically relevant in patients who achieve the LDL-C goal of $<70 \mathrm{mg} / \mathrm{dL}$ for very-high-risk patients. Analysis of the TNT data showed that as HDL-C values increased from quintile 1 to quintile 5, there were significant reductions in the occurrence of major CV events in this cohort of statin-treated patients with LDL-C $<70 \mathrm{mg} / \mathrm{dL}$. Notably, the risk of major $\mathrm{CV}$ events was 39\% lower among patients in the highest HDL-C quintile (HDL-C $\geq 55$ $\mathrm{mg} / \mathrm{dL})$ compared with patients in the lowest HDL-C quintile $(<37 \mathrm{mg} / \mathrm{dL})$. The finding that HDL-C remained a significant predictor of major $C V$ events in patients with verylow LDL-C supports the clinical relevance of HDL-C as a therapeutic target. Statintreated patients who achieve a low LDL-C goal still have a substantial residual risk that can perhaps be reduced by increasing the HDL-C concentration. 
ties of SCAR-BI gene and/or SR-BI protein may modify cholesterol levels, in particular HDL, with different incidence of atherosclerosis and CVD. ${ }^{25}$

Over expression of SR-BI reduces HDLC/ApoA-I plasma concentrations because of accelerated clearance. In mice, hepatic SR-BI over expression resulted in increased macrophage RCT (despite reduced plasma HDL-C levels) while SR-BI deficiency was associated with markedly increased atherosclerosis, reduced RCT, lipid deposition and atherosclerosis in the aorta despite increased HDL-C levels. It seems to exist an inverse relation between hepatic SR-BI expression and atherosclerosis. ${ }^{25}$ Different studies in SR-BI deficient mice on the of $\mathrm{ApoE}^{-/-}$or either $\mathrm{LDLR}^{-/}$ background results in early and markedly accelerated atherosclerosis and mortality. Moreover, bone marrow transplantation from SR-BI-deficient mice into $\mathrm{LDLR}^{-/}$or $\mathrm{ApoE}^{-/-}$ mice increases atherosclerosis, consistent with a protective role of macrophage SR-BI. ${ }^{26}$ In murine hepatocytes expression of mutant SR-BI reduced to half the cholesterol uptake from HDL compared to hepatocytes expressing wild-type SR-BI. In particular, carriers of the P297S mutation have increased HDL-C levels and a reduced capacity for cholesterol efflux from macrophages, although the carotid artery intima-media thickness was similar in carriers and in non-carriers. Platelets from carriers have increased non-esterified cholesterol content and impaired function. ${ }^{27}$ However, the P297S is not the only known mutation. Two polymorphisms of the $S R$-BI gene promoter have been identified. One is an 11-bp deletion at the transcription start site, corresponding to a specific protein binding site (Sp1) that prevents binding of $\mathrm{Sp} 1$, thus reducing transcriptional activities. It is associated with significant increase in HDL-C levels and explained $\approx 0.5 \%$ of the variation in HDL-C levels in this population. ${ }^{27}$ The other mutation is a C T substitution at position -142 .

Some studies have also described, in addition, mutations that affect in particular female suggesting that the genetic basis of dyslipidemias and the cause of coronary disease can varies between men and women. In particular, combined genotypes for a silent variant in exon 8 and the intron 5 polymorphisms are strongly associated with TG:HDL-C ratio, an important risk factor for atherosclerosis and metabolic syndrome. Interestingly, the same associations were not found in men. ${ }^{28}$

Moreover, SR-BI expression may be modified by alterations affecting other stages of the RCT. Very interesting is the relation between ApoA-I and SR-BI, in particular ApoA-I Milano $\left(\mathrm{ApoA}-\mathrm{I}_{\mathrm{M}}\right)$. It has been reported that recombinant $\mathrm{ApoA}-\mathrm{I}_{\mathrm{M}}$ administration involves an important reduction in the hepatic and aortic cholesterol content without altering plasma levels..$^{29}$ This effect was associated with an upregulation of vessel wall $\mathrm{ABCA}-1$, as well as hepatic and arterial-wall SR-BI up-regulation.

\section{ApoA-I}

ApoA-I is the major protein of HDLs and it is the key player in removing cholesterol from the macrophages/foam cells in the vessel wall. ${ }^{2}$ The structure of ApoA-I has been subject of intense study because elevated levels of HDLs correlate strongly with a reduced risk for atherosclerosis. ${ }^{3}$ In addition to its ability to solubilize lipids, ApoA-I serves as the major activator of LCAT and promotes cellular cholesterol efflux. $^{2}$

Two naturally occurring human mutations of ApoA-I (arginine to cysteine substitution), have been identified. In ApoA-I $\mathrm{I}_{\mathrm{M}}$ this mutation occurs at residue 173, whereas in ApoA-I Paris $\left(\right.$ apoA- $\left.I_{p}\right)$ it occurs at residue 151 . These mutations are interesting because wild-type ApoA-I $\left(\right.$ ApoA- $\left.\mathrm{I}_{\mathrm{wt}}\right)$ does not contain any cysteine residue. ${ }^{29}$ Paradoxically, heterozygous individuals for either mutation are at a reduced risk for atherosclerosis despite low HDL-C levels. ${ }^{30}$ Reconstituted HDLs containing homodimers of either ApoA- $\mathrm{I}_{\mathrm{M}}$ or ApoA-I $\mathrm{I}_{\mathrm{p}}$ were similar to HDLs containing ApoA- $\mathrm{I}_{\mathrm{wt}}$ in their ability to clear dimyristoyl-phosphatidyl-choline emulsions, to promote cholesterol efflux ${ }^{30}$ and to activate LCAT, although this ability is at a decreased efficiency compared to reconstituted HDLs containing ApoA $-\mathrm{I}_{\mathrm{wt}}{ }^{2}$

Furthermore, HDLs containing $\mathrm{ApoA}-\mathrm{I}_{\mathrm{M}}$ or ApoA- $I_{p}$ form either homodimers of ApoA- $I_{w t}$ or heterodimers of ApoA- $I_{w t}$ and ApoA-II. ApoA- $I_{M}$ forms reconstituted HDLs with two distinct diameters containing either two or four molecules of ApoA-I present as homodimers. These reconstituted HDLs are of a size comparable to the HDLs containing ApoA $-\mathrm{I}_{w t}$. ApoA- $\mathrm{I}_{\mathrm{p}}$ forms reconstituted HDLs with three distinct diameters. Despite an high atherogenic lipid profile, consisting in very low plasma HDL-C and moderate hypertriglyceridemia, ApoA $-\mathrm{I}_{\mathrm{M}}$ carriers present low cardiovascular risk as reported by Sirtori and Franceschini in a small group of people in Limone sul Garda, Italy, who shared that common lipid profile. ${ }^{31}$ This apparent paradox is explained, at least in part, by an enhanced capacity of serum from ApoA- $\mathrm{I}_{\mathrm{M}}$ carriers to promote cell cholesterol efflux through the ABCA-1 transporter and by an extremely prolonged elimination half-life due to a dimeric form that apparently enhanced the capacity to remove cholesterol, thus having a dramatic impact on cardiovascular risk. ${ }^{31}$

The efficiency of ApoA- $\mathrm{I}_{\mathrm{M}}$ to remove cholesterol and reduce pre-existing atherosclerotic lesion has been highly investigated..$^{32,10,29}$ In animal models, administration of recombinant ApoA $-I_{M}$ results in a reduced plaque size, lower lipid and macrophage plaque content, decreased TF and MMP levels, lower inflammation profile and enhanced RCT. ${ }^{29,32}$ In humans, 5 weekly injections induced a significant plaque reduction. ${ }^{10}$ However, whether ApoA- $\mathrm{I}_{\mathrm{M}}$ is more powerful than $A p o A-I_{w t}$ is still controversial. To date, it has been proven that recombinant HDLs carrying ApoA- $\mathrm{I}_{\mathrm{M}}$ exerts greater anti-inflammatory and plaque stabilizing properties than wild-type HDLs. ${ }^{33}$

The genes coding for apolipoprotein (i.e. ApoA-I, ApoC-III, ApoA-IV and ApoA-V) are located in clusters on chromosome 11 , and some of the patients with complete deficit of ApoA-I have deletions also in more genes of this complex. Mutations in these genes, critical in the synthesis and in the catabolism of HDLs, can induce marked changes in HDL-C plasma levels. The complete ApoA-I deficiency leads to the absence of plasma HDLs with increased plasma and tissue levels of $\mathrm{FC}$, resulting in the development of corneal opacities and planar xanthomas and finally premature CVD. On the other hand, increasing ApoAI plasma levels is associated with a significant reduction of the residual cardiovascular risk. ${ }^{34}$ Over expression of functional ApoA-I/HDL can be easily achieved by gene transfer with regression of pre-existing atherosclerotic lesion. $^{9}$

\section{Lecithin-cholesterol acyltransferase}

LCAT within the discoid nascent HDLs catalyzes the transfer of 2-acyl groups from lecithin to FC generating $\mathrm{CE}$ and lysolecithin. The CE are more hydrophobic than FC and moves to the core of the lipoprotein, allowing the formation of the larger mature, spherical shaped, HDLs. LCAT is critical for normal HDL metabolism, because its absence results in the inability to generate mature HDLs with normal CE cores. A defect in LCAT function causes extremely low HDL-C/ApoA-I levels due to rapid catabolism $^{35}$ and enhances atherosclerosis. Human LCAT gene is localized on chromosome 16 and contains 6 exons, with $1.5 \mathrm{~kb}$ of coding sequence. It is primarily expressed in the liver. LCAT protein is secreted into the plasma and it resides on circulating HDLs which contain its principal activator: ApoA-I. LCAT exhibits two activities in normal plasma: $\alpha$-LCAT activity, specific for lipoproteins with $\alpha$-mobility upon electrophoresis (i.e. HDL), and $\beta$-LCAT activity, specific for pre- $\beta$ and $\beta$-migrating lipoproteins (i.e. VLDL and LDL, respectively). However, $\alpha$ - and $\beta$-LCAT activities represent 
two functional aspects of the same protein, as only one $L C A T$ gene is present in humans. ${ }^{35}$

To date, over 60 different mutations in human LCAT gene have been reported which can lead to two rare disorders: Fish-eye disease (FED) and familial LCAT deficiency (FLD) ${ }^{35}$ FED was classified as $\alpha$-LCAT deficiency whereas FLD resulted from a lack of both $\alpha$ and $\beta$-LCAT activities. LCAT deficiency causes low HDL-C levels and an excess of non-esterified cholesterol and lecithin that distributes among plasma lipoproteins and plasma membranes. FED patients have reduced LCAT activity on HDL $(\alpha$-LCAT) but near normal activity on LDL ( $\beta$-LCAT). They present the characteristic dense age-dependent corneal opacifications and HDL-C deficiency; the conserved $\beta$ LCAT activity in FED appears to prevent the development of severe clinical symptoms as described for subjects with FLD. FLD is characterized by HDL deficiency and lipid changes in both VLDL-C and LDL-C fractions. The major clinical manifestations include corneal opacification, anemia, proteinuria, and renal disease. ${ }^{35}$ In these patients renal lesions began with the deposition of lipid-like structures in the glomerular basement membrane that progressively accumulate in the mesangium and capillary subendothelium. ${ }^{36}$ FED and FLD subjects may have normal to elevated total cholesterol and triglycerides with similar low level of HDL-C. Although CVD has been reported in FLD and FED patients, ${ }^{35}$ in many cases they do not develop clinically apparent disease. The role of LCAT in the pathogenesis of atherosclerosis is still today controversial. Even it is an anti-atherogenic factor, the final effect depends by factors that modulate the RCT pathway.

\section{Cholesterylester transfer protein}

Cholesterylester transfer protein (CETP), also called plasma lipid transfer protein, facilitates the transport of $\mathrm{CE}$ and triglycerides between the lipoproteins. It works at a crucial step in RCT, collecting triglycerides from VLDL or LDL and exchanges them for CE from HDL, and vice versa. ${ }^{2}$ It may have both pro-atherogenic and anti-atherogenic properties. The pro-atherogenic activity may result from: i) decrease of RCT via the HDL/hepatic SR-B1 route, ii) reduction in overall HDL levels, potentially reducing cholesterol efflux from the arterial wall, and iii) increase in atherogenic LDL levels. On the other hand, the anti-atherogenic properties may come from an increase in indirect RCT via the LDL/hepatic LDLR route. Thus, the question of whether the sum effect of CETP activity is proor anti-atherogenic remains to be solved. Different studies are still ongoing to evaluate if CETP modulation may be a viable strategy against atherosclerosis. ${ }^{6}$

Initial evidence of a link between CETP and HDL-C levels came from the identification of mutations in the CETP gene in subjects with plasma lipid levels abnormalities. Numerous gene variants of this protein have been identified in various countries, with often conflicting phenotypic implications. In up to $2 \%$ of the general population of Japan and in $27 \%$ of people in the Omagari region is present a nucleotide substitution ( $\mathrm{G}$ to $\mathrm{A}$ ) leading to a splicing mutation of the CETP gene that results in no measurable CETP activity and markedly raised HDL-C levels. A second mutation, even more common than the first one (up to 7\% of the Japanese population) has been identified, also causing CETP protein deficiency. It occurs in exon 15 and results in a substitution at position -442 (D442G). Interestingly, homozygous individuals for D442G retain partial CETP activity and, consequently, have less markedly raised HDL-C levels. ${ }^{37}$

Because of its key role in RCT, CETP mutations can produce significant changes in lipid and lipoprotein metabolism and these changes vary between different mutations. Moreover, CETP protein deficiency may not only affect overall HDL-C levels but may also result in qualitative changes in HDL, affecting their functionality. These lipoproteins may be larger in size and CE-rich, thus with an ambiguous ability to promote cholesterol efflux from macrophages. ${ }^{38}$ Consequently, the role played by underlying CETP mutations on the susceptibility to develop CVD is complex and difficult to define. However, not all CETP gene mutations have a dramatic effect on CETP protein levels. Various single nucleotide polymorphisms (SNPs) of the CETP gene have also been reported. They are associated with only small changes in plasma CETP levels and HDL-C levels. The most well-characterized CETP SNP is Taq1B at intron 1. This mutation is associated with high CETP activity, low HDL-C levels and increased CVD risk, although this effect may to some degree be dependent on gender. $^{39}$

Another CETP gene polymorphism, the I405V, has been also investigated. The homozygosity for the $405 \mathrm{~V}$ allele is highly present in the Ashkenazi Jews (who tend to have exceptional longevity) and it is associated with reduced CETP levels and a unique lipoprotein profile, characterized by larger HDL and LDL particles, maybe responsible of the survival advantage leading to longevity. However, previous studies of the I405V CETP gene polymorphism have provided contradictory findings. ${ }^{39}$ Taking into account the lesson from CETP mutations, we may understand and explain why pharmaceutical inhibitors of CETP, such as torcetrapib and dalcetrapib, to increase HDL-C for prevention of CVD lead to ambiguous results. The failure of torcetrapib in the ILLUMINATE trial, because of an increased cardiovascular event rate and the premature termination of the dal-OUTCOMES with dalcetrapib, for a lack of clinically significant benefit despite the high HDL-C levels achieved, raised several issues about the safety of this strategy to reduce CVD. ${ }^{6}$ Treatment with torcetrapib, in particular, was associated with hypertension, maybe for an increase in plasma levels of aldosterone and corticosterone. ${ }^{40}$

More promising results seem to come from the last inhibitors of this family, the anacetrapib. ${ }^{6}$ Its use is not associated with increased adrenal steroid levels observed with torcetrapib. However large clinical trials are needed to address its safety and efficacy in CVD patients.

On the other hand, the use of CETP agonists (i.e. probucol) may also lead to clinical benefits. As shown in the FAST (Fukuoka Atherosclerosis Trial) trial, probucol treatment led to a $>80 \%$ reduction of events, compared with $40 \%$ for pravastatin. Moreover, long-term treatment with probucol has been also used to effectively manage severe familial hypercholesterolemia. ${ }^{41}$

\section{Conclusions}

Atherosclerosis is a complex disorder resulting from the interaction between genotype (that determine the individual's susceptibility) and environment (that determine the risk factors). The relative contribution of genes and environment varies from one patient to another. As discussed above, there is no unifying genetic pattern that is associated with atherosclerosis, thus some individuals suffer from hereditary impaired lipoprotein homeostasis, others from chronic inflammation or vascular calcification that may be the prevalent cause of their increased susceptibility. As shown by the ApoA$\mathrm{I}_{\mathrm{M}}$ population, not all the genetic variants result in an increased atherosclerosis development. Placing the genetic basis of the athero- and cardio-protective properties of HDL, a better understanding of HDL biology may uncover novel opportunities to enhance its function in CVD prevention. To date, genetic information is far from ready for clinical use in CVD prediction. From the personal point of view and based on the data discussed above, promising therapeutic interventions to increase good HDL are not so far (i.e. infusion of recombinant HDL and/or ApoA-I over expression), thus we should pursue in these close strategies to achieve our goal to reduce $\mathrm{CVD}$.

\section{References}

1. Yusuf S, Reddy S, Ounpuu S, Anand S. Global burden of cardiovascular diseases: Part I: General considerations, the epidemiologic transition, risk factors, and impact of urbanization. Circulation 2001;104:2746-53. 
2. Badimon JJ, Ibanez B, Cimmino G. Genesis and dynamics of atherosclerotic lesions: Implications for early detection. Cerebrovasc Dis. 2009;27:38-47.

3. Castelli WP, Garrison RJ, Wilson PW, et al. Incidence of coronary heart disease and lipoprotein cholesterol levels. The framingham study. JAMA 1986;256:2835-8.

4. Genest JJ, McNamara JR, Salem DN, Schaefer EJ. Prevalence of risk factors in men with premature coronary artery disease. Am J Cardiol 1991;67:1185-9.

5. Barter P, Gotto AM, LaRosa JC, et al. Hdl cholesterol, very low levels of Idl cholesterol, and cardiovascular events. N Engl J Med 2007;357:1301-10.

6. Shinkai H. Cholesteryl ester transfer-protein modulator and inhibitors and their potential for the treatment of cardiovascular diseases. Vasc Health Risk Manage 2012;8:323-31.

7. Braun A, Trigatti BL, Post MJ, et al. Loss of sr-bi expression leads to the early onset of occlusive atherosclerotic coronary artery disease, spontaneous myocardial infarctions, severe cardiac dysfunction, and premature death in apolipoprotein e-deficient mice. Circ Res 2002;90:270-6.

8. Badimon JJ, Badimon L, Fuster V. Regression of atherosclerotic lesions by high density lipoprotein plasma fraction in the cholesterol-fed rabbit. J Clin Invest 1990;85:1234-41.

9. Cimmino G, Giannarelli C, Chen W, et al. Adeno-associated virus serotype 8 apoa-i gene transfer reduces progression of atherosclerosis in apoe-ko mice: comparison of intramuscular and intravenous administration. J Cardiovasc Pharmacol 2011;57: 32533 .

10. Nissen SE, Tsunoda T, Tuzcu EM, et al. Effect of recombinant apoa-i milano on coronary atherosclerosis in patients with acute coronary syndromes: a randomized controlled trial. JAMA 2003;290:2292-300.

11. Kos V, Ford RC. The atp-binding cassette family: A structural perspective. Cell Mol Life Sci 2009;66:3111-26.

12. Rust S, Rosier M, Funke H, et al. Tangier disease is caused by mutations in the gene encoding atp-binding cassette transporter 1. Nat Genet 1999;22:352-5.

13. Bocchi L, Pisciotta L, Fasano T, et al. Multiple abnormally spliced abcal mrnas caused by a novel splice site mutation of abcal gene in a patient with tangier disease. Clin Chim Acta 2010;411:524-30.

14. Yvan-Charvet L, Ranalletta M, Wang N, et al. Combined deficiency of abcal and abcgl promotes foam cell accumulation and accelerates atherosclerosis in mice. J Clin Invest 2007;117:3900-8.

15. Frikke-Schmidt R, Nordestgaard BG, Schnohr P, et al. Mutation in abcal predict- ed risk of ischemic heart disease in the copenhagen city heart study population. J Am College Cardiol 2005;46:1516-20.

16. Le Lay S, Robichon C, Le Liepvre X, et al. Regulation of abcal expression and cholesterol efflux during adipose differentiation of 3t3-11 cells. J Lipid Res 2003;44:1499-507.

17. Baldan A, Gomes AV, Ping P, Edwards PA. Loss of abcg1 results in chronic pulmonary inflammation. J Immunol 2008;180:3560-8.

18. Karten B, Campenot RB, Vance DE, Vance JE. Expression of abcg1, but not abcal, correlates with cholesterol release by cerebellar astroglia. J Biol Chem 2006;281: 4049-57.

19. Kruit JK, Wijesekara N, Westwell-Roper C, et al. Loss of both abcal and abcgl results in increased disturbances in islet sterol homeostasis, inflammation, and impaired $\beta$-cell function. Diabetes 2012;61:659-64.

20. Terasaka N, Yu S, Yvan-Charvet L, et al. Abcgl and hdl protect against endothelial dysfunction in mice fed a high-cholesterol diet. J Clin Invest 2008;118:3701-13.

21. Baldan A, Tarr P, Lee R, Edwards PA. Atpbinding cassette transporter g1 and lipid homeostasis. Curr Opin Lipidol 2006;17: 227-32.

22. Ranalletta M, Wang N, Han S, et al. Decreased atherosclerosis in low-density lipoprotein receptor knockout mice transplanted with abcg1-/- bone marrow. Arterioscler Thromb Vasc Biol 2006;26: 2308-15.

23. Burgess B, Naus $K$, Chan $J$, et al. Overexpression of human abcg1 does not affect atherosclerosis in fat-fed apoe-deficient mice. Arterioscler Thromb Vasc Biol 2008;28:1731-7.

24. Yvan-Charvet L, Pagler T, Gautier EL, et al. Atp-binding cassette transporters and hdl suppress hematopoietic stem cell proliferation. Science 2010;328:1689-93.

25. Van Eck M, Twisk J, Hoekstra M, et al. Differential effects of scavenger receptor bi deficiency on lipid metabolism in cells of the arterial wall and in the liver. $\mathrm{J}$ Biol Chem 2003:278:23699-705.

26. Zhang W, Yancey PG, Su YR, et al. Inactivation of macrophage scavenger receptor class b type i promotes atherosclerotic lesion development in apolipoprotein e-deficient mice. Circulation 2003;108:2258-63.

27. Vergeer M, Korporaal SJ, Franssen R, et al. Genetic variant of the scavenger receptor bi in humans. N Engl J Med 2011;364:136-45.

28. McCarthy JJ, Lehner T, Reeves C, et al. Association of genetic variants in the hdl receptor, sr-b1, with abnormal lipids in women with coronary artery disease. J Med Genet 2003;40:453-8.

29. Cimmino G, Ibanez B, Vilahur G, et al. Upregulation of reverse cholesterol transport key players and rescue from global inflammation by apoa-i(milano). J Cell Mol Med
2009;13:3226-35.

30. Franceschini G, Calabresi L, Chiesa G, et al. Increased cholesterol efflux potential of sera from apoa-imilano carriers and transgenic mice. Arterioscler Thromb Vasc Biol 1999;19:1257-62.

31. Sirtori CR, Calabresi L, Franceschini G, et al. Cardiovascular status of carriers of the apolipoprotein a-i(milano) mutant: the Limone sul Garda study. Circulation 2001; 103:1949-54.

32. Ibanez B, Vilahur G, Cimmino G, et al. Rapid change in plaque size, composition, and molecular footprint after recombinant apolipoprotein a-i milano (etc-216) administration: Magnetic resonance imaging study in an experimental model of atherosclerosis. J Am Coll Cardiol 2008;51: 1104-9.

33. Ibanez B, Giannarelli C, Cimmino G, et al. Recombinant hdl(milano) exerts greater anti-inflammatory and plaque stabilizing properties than hdl(wild-type). Atherosclerosis 2012;220:72-7.

34. Cziraky MJ, Watson KE, Talbert RL. Targeting low hdl-cholesterol to decrease residual cardiovascular risk in the managed care setting. J Manage Care Pharmacy 2008;14:S3-28; quiz S30-21.

35. Kuivenhoven JA, Pritchard $\mathrm{H}$, Hill J, et al. The molecular pathology of lecithin:cholesterol acyltransferase (lcat) deficiency syndromes. J Lipid Res 1997;38:191-205.

36. Jimi S, Uesugi N, Saku K, et al. Possible induction of renal dysfunction in patients with lecithin:Cholesterol acyltransferase deficiency by oxidized phosphatidylcholine in glomeruli. Arterioscler Thromb Vasc Biol 1999;19:794-801.

37. Inazu A, Jiang XC, Haraki T, et al. Genetic cholesteryl ester transfer protein deficiency caused by two prevalent mutations as a major determinant of increased levels of high density lipoprotein cholesterol. J Clin Invest 1994;94:1872-82.

38. Ishigami M, Yamashita S, Sakai N, et al. Large and cholesteryl ester-rich high-density lipoproteins in cholesteryl ester transfer protein (cetp) deficiency can not protect macrophages from cholesterol accumulation induced by acetylated low-density lipoproteins. J Biochem 1994;116:257-62.

39. Boekholdt SM, Kuivenhoven JA, Hovingh GK, et al. Cetp gene variation: relation to lipid parameters and cardiovascular risk. Curr Opin Lipidol 2004;15:393-8.

40. Forrest MJ, Bloomfield D, Briscoe RJ, et al. Torcetrapib-induced blood pressure elevation is independent of cetp inhibition and is accompanied by increased circulating levels of aldosterone. Br J Pharmacol 2008;154: 1465-73.

41. Yamashita S, Matsuzawa Y. Where are we with probucol: a new life for an old drug? Atherosclerosis 2009;207:16-23. 